Nervenarzt 2009 $\cdot 80: 1356-1356$

DOI 10.1007/s00115-009-2886-y

Online publiziert: 28. Oktober 2009

๑) Springer Medizin Verlag 2009

\author{
M. Dümpelmann \\ Klinische Psycho- und Soziotherapie, Asklepios Fachklinikum Tiefenbrunn, Rosdorf
}

\title{
Die Psychoanalyse soll gerettet werden
}

\section{Mit allen Mitteln?}

\section{Zum Beitrag}

RiefW, Hofmann SG (2009) Die Psychoanalyse soll gerettet werden. Mit allen Mitteln? Nervenarzt 80:593-597

\section{Leserbrief}

\section{Dümpelmann \\ Asklepios Fachklinikum \\ Tiefenbrunn, Rosdorf}

Rief und Hofmann beziehen sich unter diesem Titel auf eine 2008 in JAMA erschienene Metaanalyse von Leichsenring und Rabung [1] zur Effektivität psychodynamischer Langzeittherapie („effectiveness of long-term psychodynamic psychotherapy"). Dass diese Behandlungsform von Rief und Hofmann mit Psychoanalyse gleichgesetzt wird, ohne dass sie das begründen, ist nicht die einzige Auffälligkeit. Mit dieser Gleichsetzung wird aber auch zu belegen versucht, dass Leichsenring und Rabung die Effekte psychoanalytischer Langzeitbehandlung untersuchen, was nicht zutrifft. Und weiter werden dann auf dieser von Rief und Hofmann vorgenommenen Gleichsetzung aufbauend die Einschlusskriterien der Metaanalyse negativ bewertet. Auch in der weiteren Argumentation, mit der Rief und Hofmann ihre Kritik darstellen, zeigen sich erhebliche Schwächen und Fehler. Dazu pars pro toto einige Beispiele:

Der Einwand der mangelhaften Kategorisierung von Behandlungsinhalten zur Sicherung der Treatment-Integrität (S. 594), festgemacht u.a. an der Stu- die von Vinnars et al. [2], ist nicht haltbar. Die dort angewandte supportiv-expressive Therapie ist als eine psychodynamische Behandlungsform bekannt und beschrieben [3].

Auch der Vorwurf des unreflektierten Einschließens von Beobachtungsstudien in die Metaanalyse (S. 593) trifft nicht zu. Bei Leichsenring und Rabung werden Beobachtungsstudien und RCTs explizit erst einmal vorab getrennt betrachtet und auch explizit vorab miteinander verglichen, bevor die Ergebnisse beider Studientypen zur Basis von Aussagen gemacht werden (S. 1559).

Und weiter ist z. B. die Kritik, dass die Stabilität der Behandlungseffekte nicht untersucht worden sei (S. 596), unverständlich, weil dazu mehrfach Stellung genommen wird (S. 1553, Tabelle 2, 3 und 4).

Soweit zu den Argumenten von Rief und Hofmann, wie sie sich in einem Rahmen von Wissenschaftlichkeit aufgreifen, beantworten und auch erwidern lassen.

Ihre Arbeit kann aber nicht allein so betrachtet werden, enthält sie doch eine Fülle von Aussagen, die auch bei bestem Willen als polemisch und unterstellend, z.T. auch als diffamierend zu qualifizieren sind. Das beginnt mit dem Verweis auf die große Resonanz der Metaanalyse in den „Printmedien“ (S. 593), geht weiter mit dem Hinweis, dass Leichsenring und Rabung die Übereinstimmung ihrer Ergebnisse mit denen psychoanalytischer Langzeittherapie "suggerieren“ (S. 594) und gipfelt in der Unterstellung, den Einschluss von Beobachtungsstudien „offensichtlich gezielt ausgenützt“ (S. 596) zu haben. Auch die Unvoreingenommen- heit der Reviewer des JAMA wird mit der Wortwahl „Meinung" (S. 593) bezweifelt.

Als Fazit lässt sich festhalten, dass die wünschenswerte kritische wissenschaftliche Auseinandersetzung mit der für die Versorgung relevanten Methode der psychodynamischen Langzeitbehandlung hier und so scheitert, an den vorgebrachten Argumenten wie auch an tendenziellen Bemerkungen und Schlussbildungen, die in einem solchen Text fehl am Platz sind. Dass er so in Der Nervenarzt veröffentlicht wurde, verwundert.

\section{Korrespondenzadresse \\ Dr. M. Dümpelmann \\ Klinische Psycho- und Soziotherapie, Asklepios Fachklinikum Tiefenbrunn 37124 Rosdorf \\ m.duempelmann@asklepios.com}

\section{Literatur}

1. Leichsenring F, Rabung S (2008) Effectiveness of long-term psychodynamic psychotherapy. A meta-analysis. JAMA 300:1551-1564

2. Vinnars B et al (2005) Manualized supportive-expressive psychotherapy versus nonmanualized community-delivered psychodynamic therapy for patients with personality disorders: Bridging Efficacy and Effectiveness. J Am Psychoanal Assoc 162(10):1933-1940

3. Luborsky L (1984) Principles of psychoanalytic psychotherapy. Manual for supportive-expressive treatment. Basic Books, New York 